\title{
TENDENCIA Y CARACTERÍSTICAS DE LOS MANUSCRITOS RECIBIDOS Y RECHAZADOS EN LA REVISTA PERUANA DE MEDICINA EXPERIMENTAL Y SALUD PÚBLICA ENTRE 2011 Y 2017
}

\author{
Hugo Arroyo-Hernández $z^{1, a}$, Bertha Huarez ${ }^{1, b}$
}

\begin{abstract}
RESUMEN
Con el objetivo de determinar la tendencia y características de los manuscritos recibidos y rechazados en la Revista Peruana de Medicina Experimental y Salud Pública (RPMESP) se analizaron los manuscritos que siguieron el proceso editorial del 2011 a 2017 según tipo de manuscrito, características del autor corresponsal y decisiones de rechazo. Se encontró un incremento de manuscritos recibidos de 283 en el 2011 a 495 en el 2017. Los autores corresponsales del extranjero, de provincias del Perú, y del género femenino presentaron un incremento en la contribución total de manuscritos, así como de originales. El promedio global de rechazo fue de $63,1 \%$, siendo $55,8 \%$ en el 2011 y $74,7 \%$ en el 2017. La no aceptación de los manuscritos originales antes de ser enviados a revisión por pares fue principalmente por deficiencias en el estilo y formato $(51,5 \%)$. Se evidencia un incremento en la recepción y rechazo de manuscritos, con diferencias según características del autor corresponsal.
\end{abstract}

Palabras clave: Manuscritos Médicos como Asunto; Revisión por Expertos; Perú (fuente: DeCS BIREME).

\section{TREND AND CHARACTERISTICS OF MANUSCRIPTS RECEIVED AND REJECTED IN THE PERUVIAN JOURNAL OF EXPERIMENTAL MEDICINE AND PUBLIC HEALTH BETWEEN 2011 AND 2017}

\begin{abstract}
In order to determine the trend and characteristics of the manuscripts received and rejected in the Peruvian Journal of Experimental Medicine and Public Health (RPMESP), the manuscripts that followed the editorial process from 2011 to 2017 were analyzed, according to the type of manuscript, characteristics of the corresponding author, and rejection decisions. An increase in manuscripts received was found, from 283 in 2011 to 495 in 2017. Corresponding authors from abroad, from the provinces of Peru, and females presented an increase in the total contribution of manuscripts, as well as originals. The global average rejection was $63.1 \%: 55.8 \%$ in 2011 and $74.7 \%$ in 2017 . The non-acceptance of original manuscripts prior to peer review was mainly due to deficiencies in style and format (51.5\%). There is an increase in the reception of manuscripts, with differences according to the characteristics of the corresponding author.
\end{abstract}

Keywords: Medical manuscripts as subject, Peer review, Peru (source: MeSH NLM).

\section{INTRODUCCIÓN}

Las revistas científicas generan diversos indicadores, en especial de sus artículos publicados. Sin embargo, se conoce poco sobre la gestión de los manuscritos antes de su publicación, esta información permite conocer los procesos de selección de las revistas e identificar su crecimiento.

La Revista Peruana de Medicina Experimental y Salud Pública (RPMESP) desde el 2010 se encuentra indizada en bases de datos de cobertura internacional (1) y ha

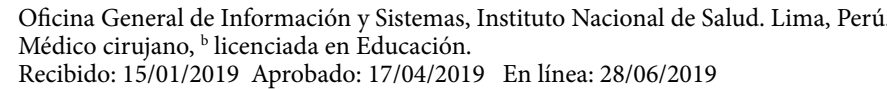


incrementado su promedio de artículos publicados ${ }^{(2,3)}$. Esto hace necesario evaluar la demanda obtenida en manuscritos recibidos y las características de sus contribuyentes. Asimismo, el análisis de manuscritos rechazados permite identificar la disposición de manuscritos que se tienen para publicar y garantizar su continuidad ${ }^{(4)}$.

La proporción de rechazo se ha considerado además como un referente de eficiencia del proceso editorial de las revistas científicas, al permitir descartar los manuscritos que no cumplan requerimientos de calidad y correctas formas de redacción e investigación ${ }^{(5,6)}$. Por lo planteado, el objetivo de esta investigación fue evaluar la tendencia y características de los manuscritos recibidos y rechazados en la RPMESP durante 2011-2017.

\section{EL ESTUDIO}

\section{DISEÑO Y RECOLECCIÓN DE DATOS}

Se revisaron los registros de los manuscritos recibidos en las diferentes secciones de la RPMESP y que siguieron el proceso editorial del 1 de enero de 2011 a 31 de diciembre de 2017. El registro de los manuscritos que ingresan al proceso editorial es generado y actualizado en una base de datos electrónica como parte de las funciones de una asistente editorial y utilizada para la elaboración de las actas con decisiones sobre los manuscritos. El proceso editorial es uniforme y realizado por los editores de la revista y revisado por el director, editor general y editor científico. Se excluyeron dos manuscritos que se encontraron publicados en otra revista y 30 que fueron retirados del proceso editorial por solicitud de los autores.

\section{PROCEDIMIENTOS}

La evaluación de los manuscritos recibidos se realiza por el comité editor, quien decide si es aceptado y se encuentra en condiciones de ser revisado por pares, o no es aceptado y se envía los motivos a los autores. Los manuscritos originales, reportes de casos, revisión, especial y simposio aceptados son evaluados por revisores y sólo las cartas al editor, galería fotográfica e historia fueron evaluadas para su aceptación y publicación por el comité editor.

Los motivos de no aceptación de los manuscritos, se clasificaron en: a. Deficiencias en estilo y formato, debido a una incorrecta presentación o sin seguir las instrucciones para el autor; b. Metodología incorrecta, si los métodos, técnicas, procedimientos e instrumentos utilizados para recopilar los datos no son los adecuados o confiables o analizados de forma incorrecta; c. Contenido no corresponde al ámbito de la revista, si la información principal del manuscrito es de mayor interés para una revista especializada o general;y d. Falta de originalidad, si el objetivo del artículo ha sido tratado en

\section{MENSAJES CLAVE}

Motivación para realizar el estudio. Existe poca información en Latinoamérica sobre los manuscritos que ingresan y son evaluados antes de su publicación en una revista científica, información que permite conocer la oferta y el proceso que demanda la gestión de una revista.

Principales hallazgos. La Revista Peruana de Medicina Experimental y Salud Pública muestra un crecimiento en el número de manuscrito recibidos con diferencias según el género y procedencia de los autores corresponsales. No obstante, más de la mitad presentan deficiencias en el estilo y formato.

Implicancias. La tendencia creciente en el número de manuscritos recibidos podría demandar una mayor gestión editorial y selección de los manuscritos recibidos.

números anteriores de la revista, ha sido ampliamente investigado o no presenta información contributiva. No se realizó una caracterización de las principales deficiencias metodológicas reportadas por los revisores.

La calificación y observaciones de los revisores se comunica a los autores siendo la decisión de no aprobación del manuscrito, si las observaciones invalidan su publicación o si luego de remitida las observaciones a los autores no se realizó una adecuada corrección, o no se obtuvo respuesta por parte de los autores; o caso contrario se aprueba la publicación luego de responder y levantar adecuadamente a las observaciones realizadas al manuscrito.

\section{VARIABLES}

Según las secciones de la revista se consideró como originales a los artículos originales y originales breves, se evaluaron además los reportes de casos, revisión, simposio, especial y cartas al editor. Las secciones de editorial, historia de la salud pública y galería fotográfica fueron agrupados como otros. Para los autores corresponsales, quien asume la comunicación con la revista, se identificó el género (varones, mujeres) según el primer o segundo nombre y realizando una búsqueda en Internet en caso de duda; la procedencia según la filiación (Perú o extranjero) y el grado académico (bachiller/técnico/profesional o estudiante de pregrado). Se definieron las decisiones editoriales como no aceptado, si el manuscrito fue rechazado por el comité editor en una evaluación inicial o como no aprobado si el manuscrito fue rechazado luego de la revisión por pares.

\section{ANÁLISIS}

Se describen las frecuencias y porcentajes de los manuscritos recibidos por años según secciones de la revista, características del autor corresponsal y para manuscritos originales, la proporción según los tipos de rechazo se calculó del total de manuscritos recibidos por 
cada sección. Los análisis se realizaron en el programa estadístico Stata $®$ versión 14.0 (Stata Corp, TX, USA).

\section{CONSIDERACIONES ÉTICAS}

El estudio tuvo la aprobación del director de la RPMESP. Se guardó la confidencialidad y protección de los datos de los autores y manuscritos, no se analizó ni compartió información que permitan generar ventaja o desventaja en el proceso editorial sobre el contenido de los manuscritos.

\section{RESULTADOS}

Se analizaron un total de 2690 manuscritos recibidos con una distribución de 283 el 2011 a 495 el 2017, siendo constante el número de manuscritos aprobados para su publicación (Figura 1).

El $52,3 \%$ del total de manuscritos fueron originales con una distribución de $151(53,4 \%)$ el 2011 a 275 (55,6\%) en el 2017 , las cartas al editor muestran un crecimiento de $52(18,4 \%)$ el 2011 a $110(22,2 \%)$ en el 2017 , los reportes de casos un incremento de $16(5,7 \%)$ en el 2011 a $36(7,3 \%)$ en 2017 , los manuscritos de simposio y especiales tuvieron una proporción total de 9,9\% sin variación en los años al igual que las revisiones, mientras que otros tipos de manuscritos presentaron una disminución en su recepción (Tabla 1).

Según los autores corresponsales para el total de manuscritos el $79,7 \%$ fueron peruanos, con un incremento de corresponsales del extranjero de 45 $(15,9 \%)$ en el 2011 a $106(21,4 \%)$ el 2017 , el $84,0 \%$ de los corresponsales peruanos fueron de Lima con un incremento de corresponsales de otras regiones, existe un incremento de corresponsales mujeres de 63 $(22,3 \%)$ en el 2011 a $180(36,4 \%)$ en el 2017 (Tabla 2$).$

Al evaluar los manuscritos originales existe un incremento de corresponsales extranjeros de 19 (12,6\%) en el 2011 a 77

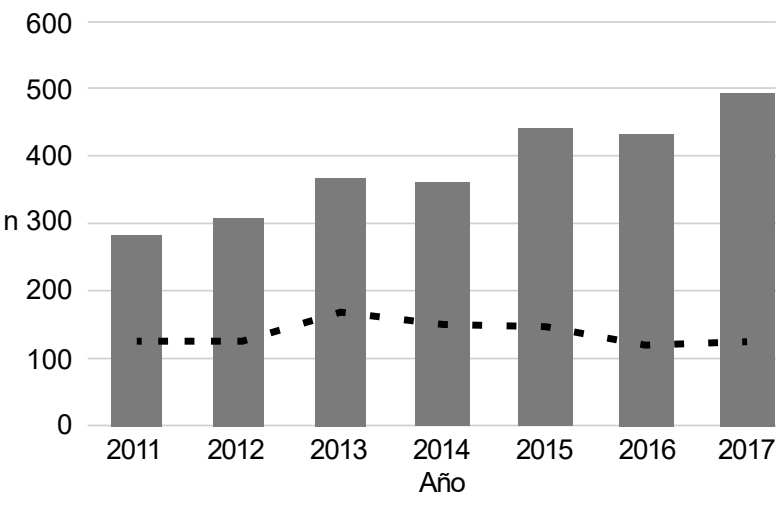

Manuscritos recibidos - - Manuscritos aprobados para publicar

Figura 1. Frecuencia de manuscritos recibidos en la Revista Peruana de Medicina Experimental y Salud Pública durante 2011 a 2017

(28,0\%) en el 2017, según los corresponsales con filiación peruana se evidencia un incremento de corresponsales de otras regiones del Perú de $20(15,2 \%)$ en el 2011 a 58 $(29,3 \%)$ en el 2017 , el $64,3 \%$ de manuscritos originales fueron de corresponsales varones, encontrando un incremento de corresponsales mujeres de 34 (22,5\%) en el 2011 a $115(41,8 \%)$ en el 2017 , el $90,2 \%$ del total de originales fueron de profesionales (Tabla 2 ).

Para el total de manuscritos el rechazo se incrementó de 158 (55,8\%) el 2011 a 370 (74,7\%) en 2017 (Figura 2); según el tipo de rechazo el $52 \%$ (42,8\% a $65,5 \%)$ de manuscritos no fue aceptado por el comité editor y $12,3 \%$ (9,3\%, a $15,5 \%)$ no fue aprobado luego de revisión por pares. El rechazo de manuscritos originales fue de $71,9 \%(59,7 \%$, a $83,3 \%)$, para reporte de casos $71,4 \%(55,6 \%$, a $85,3 \%)$, revisiones $64,1 \%$ $(41,7 \%$, a $84,2 \%)$, cartas al editor $64,5 \%(46,2 \%$, a $80,0 \%)$ y para otro tipo de manuscritos $34,5 \%(18,2 \%$, a $41,9 \%)$ (Figura 3).

Tabla 1. Tipos de manuscritos recibidos en la Revista Peruana de Medicina Experimental y Salud Pública durante 2011-2017.

\begin{tabular}{|c|c|c|c|c|c|c|c|c|c|c|c|c|}
\hline \multirow[b]{2}{*}{ Año } & \multicolumn{2}{|c|}{ Originales } & \multicolumn{2}{|c|}{$\begin{array}{l}\text { Reporte de } \\
\text { casos }\end{array}$} & \multicolumn{2}{|c|}{ Revisión } & \multicolumn{2}{|c|}{$\begin{array}{c}\text { Simposio/ } \\
\text { Especial }\end{array}$} & \multicolumn{2}{|c|}{$\begin{array}{c}\text { Cartas al } \\
\text { editor }\end{array}$} & \multicolumn{2}{|c|}{ Otros } \\
\hline & $\mathbf{n}$ & $\%$ & $\mathbf{n}$ & $\%$ & $\mathbf{n}$ & $\%$ & $\mathbf{n}$ & $\%$ & $\mathbf{n}$ & $\%$ & $\mathbf{n}$ & $\%$ \\
\hline 2011 & 151 & 53,4 & 16 & 5,7 & 12 & 4,2 & 36 & 12,7 & 52 & 18,4 & 16 & 5,7 \\
\hline 2012 & 151 & 48,9 & 18 & 5,8 & 14 & 4,5 & 35 & 11,3 & 69 & 22,3 & 22 & 7,1 \\
\hline 2013 & 171 & 46,3 & 22 & 6,0 & 13 & 3,5 & 53 & 14,4 & 92 & 24,9 & 18 & 4,9 \\
\hline 2014 & 181 & 50,4 & 30 & 8,4 & 30 & 8,4 & 22 & 6,1 & 82 & 22,8 & 14 & 3,9 \\
\hline 2015 & 250 & 56,6 & 26 & 5,9 & 16 & 3,6 & 41 & 9,3 & 100 & 22,6 & 9 & 2,0 \\
\hline 2016 & 229 & 52,9 & 34 & 7,9 & 19 & 4,4 & 43 & 9,9 & 95 & 21,9 & 13 & 3,0 \\
\hline 2017 & 275 & 55,6 & 36 & 7,3 & 24 & 4,8 & 37 & 7,5 & 110 & 22,2 & 13 & 2,6 \\
\hline Total & 1408 & 52,3 & 182 & 6,8 & 128 & 4,8 & 267 & 9,9 & 600 & 22,3 & 105 & 3,9 \\
\hline
\end{tabular}


Tabla 2. Manuscritos totales y originales recibidos en la Revista Peruana de Medicina Experimental y Salud Pública según características de los autores corresponsales durante 2011-2017.

\begin{tabular}{|c|c|c|c|c|c|c|c|c|c|c|c|c|c|c|c|c|}
\hline \multirow[b]{3}{*}{ Tipo / Año } & \multicolumn{4}{|c|}{ Procedencia } & \multicolumn{4}{|c|}{ Corresponsales en Perú } & \multicolumn{4}{|c|}{ Género } & \multicolumn{4}{|c|}{ Grado académico } \\
\hline & \multicolumn{2}{|c|}{ Perú } & \multicolumn{2}{|c|}{ Extranjero } & \multicolumn{2}{|c|}{ Lima } & \multicolumn{2}{|c|}{$\begin{array}{l}\text { Otras } \\
\text { regiones }\end{array}$} & \multicolumn{2}{|c|}{ Varones } & \multicolumn{2}{|c|}{ Mujeres } & \multicolumn{2}{|c|}{ Profesional } & \multicolumn{2}{|c|}{ Estudiante } \\
\hline & $\mathrm{n}$ & $\%$ & $\mathrm{n}$ & $\%$ & $\mathrm{n}$ & $\%$ & $\mathrm{n}$ & $\%$ & $\mathbf{n}$ & $\%$ & $\mathrm{n}$ & $\%$ & $\mathrm{n}$ & $\%$ & $\mathrm{n}$ & $\%$ \\
\hline \multicolumn{17}{|l|}{$\begin{array}{l}\text { Total de } \\
\text { manuscritos }\end{array}$} \\
\hline 2011 & 238 & 84,1 & 45 & 15,9 & 208 & 87,4 & 30 & 12,6 & 220 & 77,7 & 63 & 22,3 & 261 & 92,2 & 22 & 7,8 \\
\hline 2012 & 253 & 81,9 & 56 & 18,1 & 230 & 90,9 & 23 & 9,1 & 228 & 73,8 & 81 & 26,2 & 267 & 86,4 & 42 & 13,6 \\
\hline 2013 & 292 & 79,1 & 77 & 20,9 & 262 & 89,7 & 30 & 10,3 & 271 & 73,4 & 98 & 26,6 & 317 & 85,9 & 52 & 14,1 \\
\hline 2014 & 282 & 78,6 & 77 & 21,4 & 247 & 87,6 & 35 & 12,4 & 238 & 66,3 & 121 & 33,7 & 309 & 86,1 & 50 & 13,9 \\
\hline 2015 & 350 & 79,2 & 92 & 20,8 & 308 & 88,0 & 42 & 12,0 & 299 & 67,6 & 142 & 32,1 & 366 & 82,8 & 76 & 17,2 \\
\hline 2016 & 340 & 78,5 & 93 & 21,5 & 252 & 74,1 & 88 & 25,9 & 287 & 66,3 & 146 & 33,7 & 369 & 85,2 & 64 & 14,8 \\
\hline 2017 & 389 & 78,6 & 106 & 21,4 & 295 & 75,8 & 94 & 24,2 & 315 & 63,6 & 180 & 36,4 & 425 & 85,9 & 70 & 14,1 \\
\hline Total & 2144 & 79,7 & 546 & 20,3 & 1802 & 84,0 & 342 & 16,0 & 1858 & 69,1 & 831 & 30,9 & 2314 & 86,0 & 376 & 14,0 \\
\hline \multicolumn{17}{|l|}{$\begin{array}{l}\text { Manuscritos } \\
\text { originales }\end{array}$} \\
\hline 2011 & 132 & 87,4 & 19 & 12,6 & 112 & 84,8 & 20 & 15,2 & 117 & 77,5 & 34 & 22,5 & 140 & 92,7 & 11 & 7,3 \\
\hline 2012 & 121 & 80,1 & 30 & 19,9 & 106 & 87,6 & 15 & 12,4 & 109 & 72,2 & 42 & 27,8 & 142 & 94,0 & 9 & 6,0 \\
\hline 2013 & 127 & 74,3 & 44 & 25,7 & 111 & 87,4 & 16 & 12,6 & 113 & 66,1 & 58 & 33,9 & 152 & 88,9 & 19 & 11,1 \\
\hline 2014 & 138 & 76,2 & 43 & 23,8 & 121 & 87,7 & 17 & 12,3 & 114 & 63,0 & 67 & 37,0 & 168 & 92,8 & 13 & 7,2 \\
\hline 2015 & 191 & 76,4 & 59 & 23,6 & 161 & 84,3 & 30 & 15,7 & 155 & 62,0 & 95 & 38,0 & 214 & 85,6 & 36 & 14,4 \\
\hline 2016 & 162 & 70,7 & 67 & 29,3 & 118 & 72,8 & 44 & 27,2 & 138 & 60,3 & 91 & 39,7 & 212 & 92,6 & 17 & 7,4 \\
\hline 2017 & 198 & 72,0 & 77 & 28,0 & 140 & 70,7 & 58 & 29,3 & 160 & 58,2 & 115 & 41,8 & 242 & 88,0 & 33 & 12,0 \\
\hline Total & 1069 & 75,9 & 339 & 24,1 & 869 & 81,3 & 200 & 18,7 & 906 & 64,3 & 502 & 35,7 & 1270 & 90,2 & 138 & 9,8 \\
\hline
\end{tabular}

Los motivos de no aceptación de los manuscritos originales por el comité editor fueron en el 51,5\% (720/1399) por deficiencias en el estilo y formato, seguido del $28 \%$ (392/1399) por metodología incorrecta, 10,7\% (150/1399) por contenido del manuscrito fuera del ámbito de la revista y $9,8 \%(137 / 1399)$ por falta de originalidad.

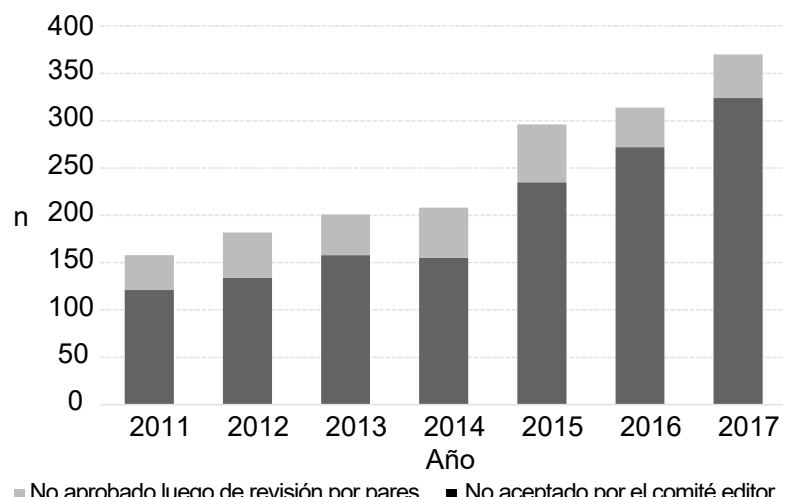

Figura 2. Frecuencia del total de manuscritos según tipo de rechazo en la Revista Peruana de Medicina Experimental y Salud Pública durante 2011 a 2017.

\section{DISCUSIÓN}

Los hallazgos evidencian un incremento en la recepción de manuscritos en la RPMESP, siendo mayor las contribuciones originales, cartas al editor y reporte de casos. Existe además un incremento en los manuscritos de corresponsales extranjeros, de provincias del Perú y del género femenino, tanto para el total de manuscritos como para la sección originales. Sin embargo, el proceso de selección editorial se hace más riguroso, pero manteniendo una frecuencia constante de manuscritos publicados.

Al evaluar las características de los autores corresponsales, el incremento de corresponsales mujeres se presentó en el total de manuscritos recibidos como en originales, a diferencia de lo reportado en revistas peruanas indexadas a SciELO donde el porcentaje de corresponsales mujeres no presenta mayor incremento durante 2010 y $2015^{(7)}$. Esta evaluación es cada vez más necesaria de explorar en nuestra región, donde la participación de mujeres en publicación científica es aún baja.

La tendencia creciente en la recepción del total de manuscritos, así como en originales de corresponsales del extranjero, refuerza el interés generado por la RPMESP en 
Total de manuscritos

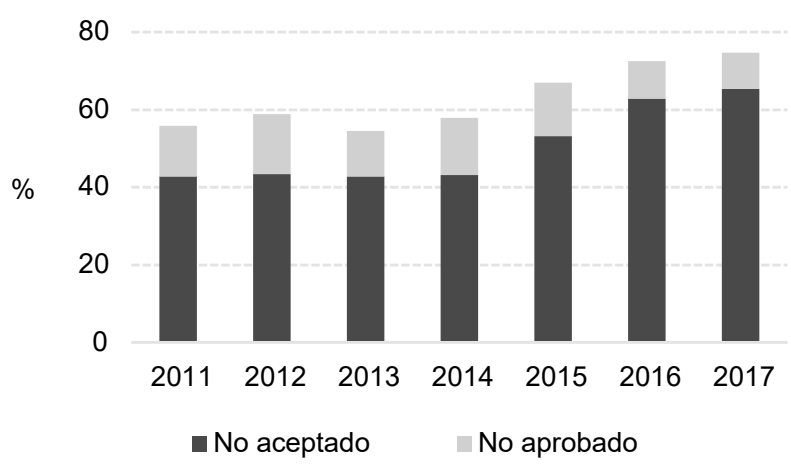

Reporte de casos 100

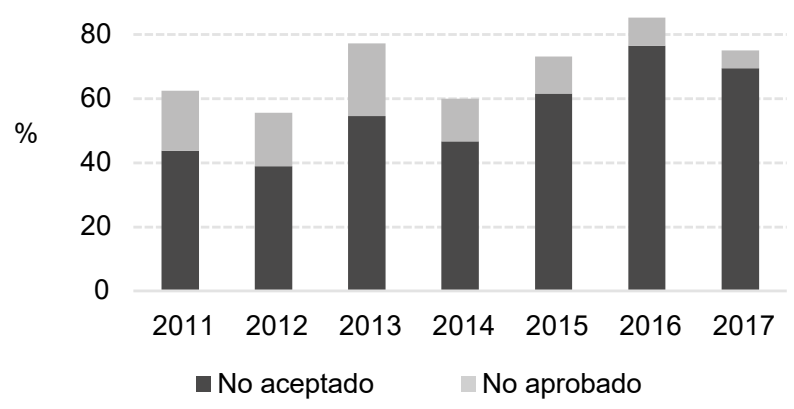

Cartas al editor

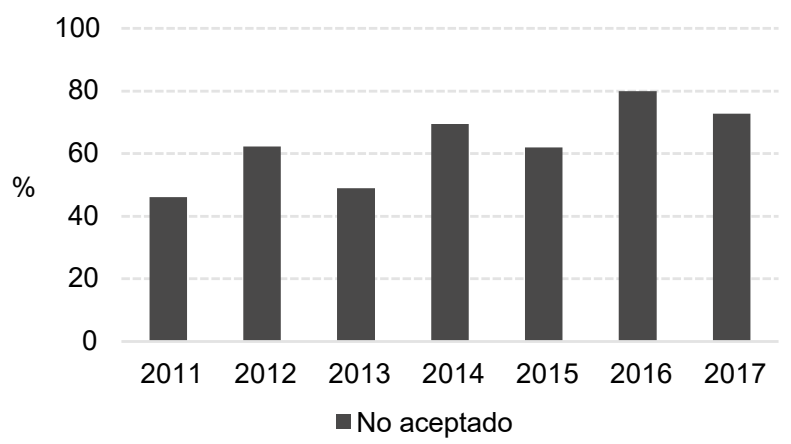

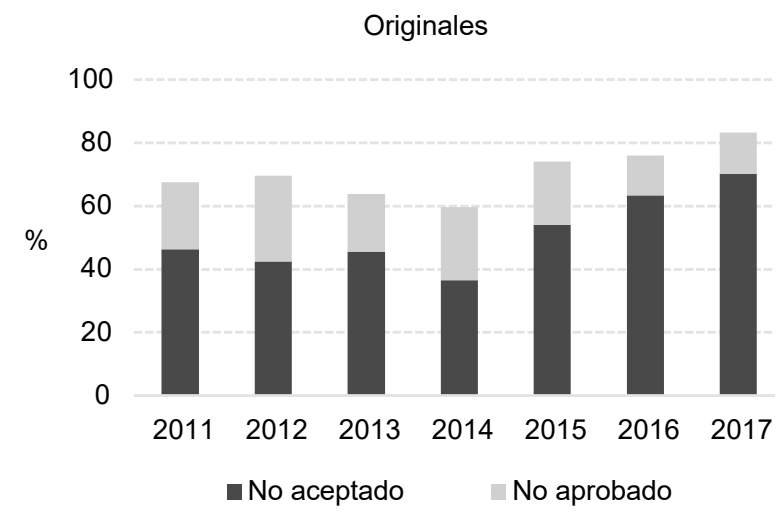
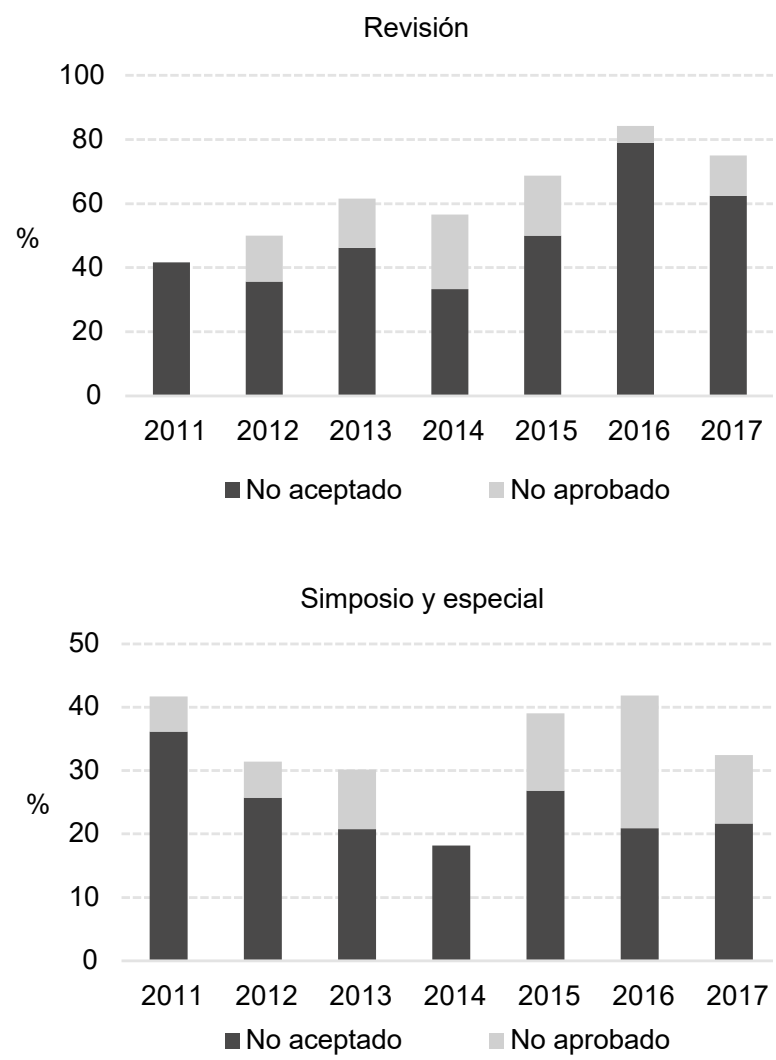

Figura 3. Proporción del rechazo según tipo de manuscritos recibidos en la Revista Peruana de Medicina Experimental y Salud Pública del 2011 al 2017.

su visibilidad internacional ${ }^{(3)}$. Al evaluar las contribuciones de originales por corresponsales peruanos la tendencia es mayor para corresponsales de provincias y podría mostrar un proceso de desarrollo descentralizado de la investigación y su interés por la publicación. El incremento en las contribuciones de corresponsales de pregrado, en especial en el total de manuscritos sugiere que esta contribución podría ser mayor para cartas al editor y reporte de casos.

Diversos factores podrían explicar el creciente interés del envío de manuscritos a la RPMESP, uno de ellos es la visibilidad desde su ingreso a bases de datos internacionales, así como mantener su periodicidad ${ }^{(1,2)}$, considerando la existencia de pocas revistas peruanas indizadas en bases de datos internacionales. Otro factor estaría relacionado con una mayor motivación para la publicación científica por universidades e instituciones en el Perú en los últimos años ${ }^{(8-11)}$.

La RPMESP publicaba un gran número de cartas al editor en comparación con otras revistas peruanas ${ }^{(12)}$. Si bien, su publicación es decisión del comité editor, en los últimos años se busca dar preferencia a las cartas científicas, y que son evaluadas por un revisor. Asimismo, existe un 
incremento de reporte de casos, aunque es una sección con pocas publicaciones en la revista ${ }^{(2)}$, por su contenido podrían ser de mayor interés en revistas clínicas o médicas generales ${ }^{(13)}$.

Otras revistas como la Revista de Saúde Pública a inicios del 2010, recibía, más de mil manuscritos por año, siendo su frecuencia de rechazo del $87 \%{ }^{(14)}$, en la Revista Panamericana de Salud Pública se menciona que los manuscritos recibidos se incrementaron de 717 en el 2014 a 1041 en el 2017 con un rechazo del 85\% (15), la Iran Journal of Public Health muestra que durante el 2016 de 1970 manuscritos recibidos el $72 \%$ fueron rechazados ${ }^{(16)}$, valores de recepción y de rechazo superiores al encontrado en el presente estudio. La revista española Gaceta Sanitaria menciona que durante el 2017 recibió 561 manuscritos, con una tendencia a incrementarse en los últimos años y una no aceptación editorial, sin pasar a revisión por pares, del $62 \%{ }^{(17)}$, valor cercano al presente estudio.

Una evaluación realizada el 2015 por SciELO Brasil, a través de una consulta realizada a 85 revistas en ciencias de la salud, muestra una media de 393 manuscritos recibidos con un promedio de rechazo del $77,1 \%{ }^{(18)}$, proporción de rechazo superior al presente estudio. En consecuencia, al igual que otras revistas internacionales la RPMESP se enfrenta a la decisión de no poder publicar todos los manuscritos recibidos. Un incremento de manuscritos recibidos lleva una mayor gestión editorial, uso de sus recursos humanos y tiempo para tomar decisiones sobre los manuscritos.

Un alto porcentaje de rechazo no se debe considerar como indicador de impacto ${ }^{(19)}$. Por lo tanto, altos porcentajes de rechazo no se podría considerar como un indicador de selección de manuscritos más citables, en especial para el contexto de las revistas Latinoamericanas, muchas de ellas con limitaciones para publicar un adecuado número de artículos, que en contraparte con aquellas con una aceptable recepción de manuscritos tienen como labor más compleja, identificar las mejores propuestas previas a la evaluación por pares.
La primera evaluación de un manuscrito debe permitir que su envío a revisión por pares sea el adecuado en formato y contenido, asimismo no se podría aceptar un manuscrito por deficiencias metodológicas, siendo este uno de los motivos de rechazo más frecuentes en la revisión por pares ${ }^{(20)}$. La revista Gaceta Sanitaria menciona que cinco de cada diez rechazos se deben principalmente a que el contenido del manuscrito no era adecuado para la revista ${ }^{(17)}$, en la revista Iranian Journal of Public Health se menciona como principales motivos de rechazo, contenido fuera del ámbito de la revista, resultados deficientes, estudios locales o contenido principalmente clínico ${ }^{(16)}$. Los principales motivos de no aceptación para originales en la RPMESP podrían deberse también por inexperiencia de los autores o no seguir las instrucciones en la presentación.

Se debe señalar algunas limitaciones como el seleccionar sólo al autor corresponsal para identificar algunas características de los manuscritos. Otra limitación es la falta de una caracterización de las principales causas de rechazo de los revisores para manuscritos originales. Si bien, podría existir la duda sobre el sesgo editorial, al igual que en otras revistas las evaluaciones realizadas en la RPMESP no dependen de una sola persona.

Concluimos que el incremento de manuscritos recibidos es positivo, mostrando un interés nacional e internacional, siendo las frecuencias de rechazos aceptables, caso contrario, se estaría en escases de artículos. Los principales motivos de no aceptación demandan de los autores una mayor atención al contenido y cuidado en el formato de envío de los manuscritos.

Contribuciones de autoría: $\mathrm{HAH}$ y $\mathrm{BH}$ participaron en la concepción, diseño del artículo, recolección de resultados, análisis e interpretación de datos, redacción del artículo, revisión crítica del artículo y aprobación de la versión final.

Fuentes de financiamiento: autofinanciada.

Conflictos de interés: $\mathrm{HAH}$ y $\mathrm{BH}$ forman parte del comité editor de la RPMESP.

\section{REFERENCIAS BIBLIOGRÁFICAS}

1. Burstein Z, Mayta-Tristán P. La Revista PeruanadeMedicinaExperimentalySalud Pública ahora indizada en MEDLINE/ Index Medicus. Rev Peru Med Exp Salud Publica. 2010;27(3):312-14. doi: 10.17843/rpmesp.2010.273.1487.

2. Huamani C. Análisis de la producción, visibilidad y citación de la Revista Peruana de Medicina Experimental y Salud Pública, 2002 - 2009. Rev Peru Med Exp Salud Publica. 2010;27(3):367-72. doi: 10.17843/rpmesp.2010.273.1494.

3. Romaní F, Cabezas C. Indicadores bibliométricos de las publicaciones científicas de la Revista Peruana de Medicina ExperimentalySalud Pública,2010-2017.RevPeru
Med Exp Salud Publica. 2018;35(4):620-9. doi:10.17843/rpmesp.2018.354.3817.

4. Fuentes García S, Tápanes Galvan W, Lantigua Méndez M, Martínez Aportela E, Román Carriera JC. La evaluación editorial y el índice de rechazo en las revistas biomédicas. Rev Med Electrón. 2015;37(4):389-97. 
5. Lee KP, Schotland M, Bacchetti P, Bero LA. Association of journal quality indicators with methodological quality of clinical research articles. JAMA. 2002;287(21):2805-8. doi:10.1001/ jama.287.21.2805.

6. Altman DG, Goodman SN, Schroter S. How statistical expertise in used in medical research. JAMA. 2002;287(21):2817-20. doi:10.1001/jama.287.21.2817.

7. Centeno-Leguía D, Morales-Concha L, Lopez CE. Mujeres científicas: características y factores asociados a la primera autoría y corresponsalía en revistas peruanas indizadas a SciELO, 2010-2015. Educ Med. 2018. doi: 10.1016/j.edumed.2018.04.010

8. Arriola-Quiroz I, Curioso WH, CruzEncarnación M, Gayoso O. Characteristics and publication patterns of theses from a Peruvian medical School. Heath Info Libr J.2010;27(2):148-54. doi: 10.1111/j.14711842.2010.00878.x.

9. Taype-Rondán Á, Luque Bustamante L. Producción científica en Scopus de la Universidad de San Martín de Porres, Lima, Perú. Horiz Med. 2014;14(4):37-42.

10. Purizaca-Rosillo N, Cardoza-Jiménez K, Herrera-Añazco P. Producción científica en una universidad pública peruana beneficiaria del canon. An Fac med. 2016;77(1):73-4. doi: 10.15381/anales. v77il.11561.

11. Consejo Nacional de Ciencia, Tecnología e Innovación Tecnológica. Incentivo para la publicación efectiva de artículos científicos en revistas indizadas - 2015 [Internet]. Lima: Concytec; 2015 [citado el 22 de noviembre de 2018]. Disponible en: http://www.cienciactiva.gob.pe/images/ documentos/convocatorias/estimulos/ publicaciones-2016/bases_incentivo_2016. pdf

12. Montenegro-Idrogo JJ, Mejía-Dolores JW, Chalco-Huamán JL. Cartas al editor publicadas en revistas biomédicas peruanas indizadas en ScIELoPerú 20062013. Rev Peru Med Exp Salud Publica. 2015;32(1):104-9. doi: 10.17843/ rpmesp.2015.321.1582.

13. Romaní F, Wong P. Reporte y serie de casos en el Perú: situación de un tipo de publicación subestimada. Análisis de las revistas médicas peruanas indizadas en SciELO-Perú, 1997-2008. Rev Peru Epidemiol. 2009;13(3):1-7.

14. Antunes JL, França Júnior I, de Andrade MT, Barata Rde C, Monteiro CA. Desafios editoriais da Revista de Saúde Pública. Ciência \& Saúde Coletiva. Cien Saude Colet. 2015;20(7):1997-2006. doi: 10.1590/1413-81232015207.02672015.

15. Pan American Health Organization. Revista Panamericana de Salud Pública. Metrics [Internet]. Washington, D.C.: PAHO; c2018 [citado el 21 de noviembre de 2018]. Disponible en: https://www.paho.org/ journal/index.php?option=com_content\&view $=$ article\&id $=180$ : metrics\&Itemid=861.

16. Farhud DD. Analysis of Papers Submitted to "Iranian Journal of Public Health" during 2013-2016. Iran J Public Health. 2017;46(1):i-iii.

17. Bermúdez-Tamayo C, Hernández MN, Alguacil J, Vozmediano EB, Cantarero
D, Portiño MC, et al. Gaceta Sanitaria en 2017. Mejorando la calidad de nuestra revista. Gac Sanit. 2018;32(2):117-120. doi: 10.1016/j.gaceta.2018.02.002.

18. Packer AL, Mendonça A, Almeida F. Flujo de manuscritos y artículos procesados por las revistas SciELO Brasil en 2014 y 2015 [Internet]. Sao Paulo: SciELO en Perspectiva; 2016 [citado el 6 de diciembre de 2018]. Disponible en: https://blog.scielo.org/ es/2016/09/23/flujo-de-manuscritos$\mathrm{y}$-articulos-procesados-por-las-revistasscielo-brasil-en-2014-y-2015.

19. Rocha da Silva P. Frontiers Blog: New data debunks old beliefs: Part 2 [Internet]. Lausanne Switzerland: Frontiers; 2016 [citado el 5 de enero de 2019]. Disponible en: https://blog. frontiersin.org/2016/03/04/initialfindings-confirmed-no-significantlink-between-rejection-rate-andjournal-impact/

20. Vega FL. Errores en los manuscritos enviados a publicación: a propósito de los requerimientos. Rev Mex Pediatr. 1998;65(1):2-4.
Correspondencia: Hugo Arroyo Hernández Dirección: Instituto Nacional de Salud. Cápac Yupanqui 1400. Jesús María. Lima, Perú Correo electrónico: hugoarroyo2007@gmail.com 